\title{
Elaboración Sostenible de Harina de Chile Jalapeño (Capsicum annuum L.) Mediante Secado Solar Tecnificado
}

\section{Gustavo Antonio Pech-González}

Egresado de Ingeniería en Alimentos, División Académica Multidisciplinaria de los Ríos, Universidad Juárez Autónoma de Tabasco, México

\section{Érika Guadalupe Ceballos-Falcón}

Profesora de la Licenciatura en Administración y Maestría en Desarrollo Alimentario Sustentable. División Académica Multidisciplinaria de los Ríos, Universidad Juárez Autónoma de Tabasco, México

\section{Nicolás González-Cortés \\ Román Jiménez-Vera}

Profesor de Ingeniería en Alimentos y Maestría en Desarrollo Alimentario Sustentable. División Académica Multidisciplinaria de los Ríos, Universidad Juárez Autónoma de Tabasco, México

\section{Doi: 10.19044/esj.2018.v14n30p15 URL:http://dx.doi.org/10.19044/esj.2018.v14n30p15}

\begin{abstract}
Jalapeño pepper (Capsicum annuum L.) is a crop in protected natural areas of Tabasco, Mexico. Solar drying is a non-polluting energy that is applicable to food processing. This paper focuses on comparing the microbiological quality of dehydrated jalapeño peppers by means of three methods: direct solar (SD), electric stove (EE), and experimental solar dryer (SSE). SD drying was carried out in zinc sheet by conduction. The drying in $\mathrm{EE}$ was at a temperature of $70^{\circ} \mathrm{C}$ and the SSE has an internal temperature of $43{ }^{\circ} \mathrm{C} \pm 2{ }^{\circ} \mathrm{C}$. The dehydrated chiles were crushed in artisanal equipment, and the flour was stored in tightly closed glass containers at room temperature $\left(22-28^{\circ} \mathrm{C}\right)$. The drying time was lower in the EE $(7 \mathrm{~h})$, followed by the SSE $(8 \mathrm{~h})$ and the SD $(18 \mathrm{~h})$. With EE, the lowest microbial load was obtained and with SD, the highest was obtained. The flour obtained with the SSE presented a concentration of microorganisms within the Mexican norms: total coliforms (25 CFU / g), Staphylococcus aureus (62 CFU / g), and aerobic mesophiles (57 CFU / g). Although the drying time and the microbial load were lower with the EE, the SSE allowed the elaboration of jalapeño pepper flour with the microbiological quality required for human consumption. Technified solar
\end{abstract}


drying is a sustainable alternative for the dehydration of foods in tropical countries.

Keywords: Flour, pepper, jalapeño, technified solar drying

\section{Resumen}

El chile jalapeño (Capsicum annuum L.) es un cultivo en áreas naturales protegidas de Tabasco, México. El secado solar es una energía no contaminante aplicable al procesamiento de alimentos. El objetivo fue comparar la calidad microbiológica de harina de chiles jalapeños deshidratados mediante tres métodos: solar directo (SD), en estufa eléctrica (EE) y en secador solar experimental (SSE). El secado SD se realizó en lámina de zinc, mediante conducción; el secado en EE a una temperatura de $70{ }^{\circ} \mathrm{C}$ y el SSE, con una temperatura interior de $43^{\circ} \mathrm{C} \pm 2{ }^{\circ} \mathrm{C}$. Los chiles deshidratados se trituraron en equipo artesanal; la harina se almacenó en recipientes de vidrio herméticamente cerrados, a temperatura ambiente $\left(22-28{ }^{\circ} \mathrm{C}\right)$. El tiempo de secado fue menor en la EE (7 h), seguido del SSE ( $8 \mathrm{~h})$ y el SD (18 h). Con la EE se obtuvo la menor carga microbiana y con el SD, la mayor. La harina obtenida con el SSE presentó una concentración de microorganismos dentro de las normas mexicanas: coliformes totales (25 UFC/g), Staphylococcus aureus (62 UFC/g) y mesófilos aeróbios (57 UFC/g). Aunque el tiempo de secado y la carga microbiana fue menor con la EE, el SSE permitió elaborar harina de chile jalapeño con la calidad microbiológica requerida para el consumo humano. El secado solar tecnificado es una alternativa sustentable para la deshidratación de alimentos en países tropicales.

Palabras clave: Harina, chile jalapeño, secado solar tecnificado

\section{Introducción}

Las Áreas Naturales Protegidas (ANP) son zonas del territorio mexicano en donde los ambientes originales no han sido alterados significativamente por la actividad del ser humano o que requieren ser preservadas y restauradas. Se crean mediante un decreto presidencial y las actividades que pueden llevarse a cabo en ellas se establecen de acuerdo con la Ley General del Equilibrio Ecológico y Protección al Ambiente. La Comisión Nacional de Áreas Naturales Protegidas (CONANP) administra actualmente 176 áreas naturales de carácter federal que representan más de 25,394,779 ha (CONANP, 2016). En Tenosique, Tabasco, México, se encuentra el Área de Protección de Flora y Fauna Cañón del Usumacinta (Figura 1), donde el cultivo de chile jalapeño (Capsicum annuum L.) a pequeña escala es una de las actividades agrícolas permitidas a sus pobladores. En apoyo a su economía es necesario evaluar procesos sustentables para la 
conservación de sus productos agrícolas con la evaluación de procesos que funcionen, en su mayor parte, con energías alternativas. De ahí que surge la siguiente pregunta ¿Qué métodos sustentables pueden aplicarse para la conservación del chile jalapeño producido en un ANP?

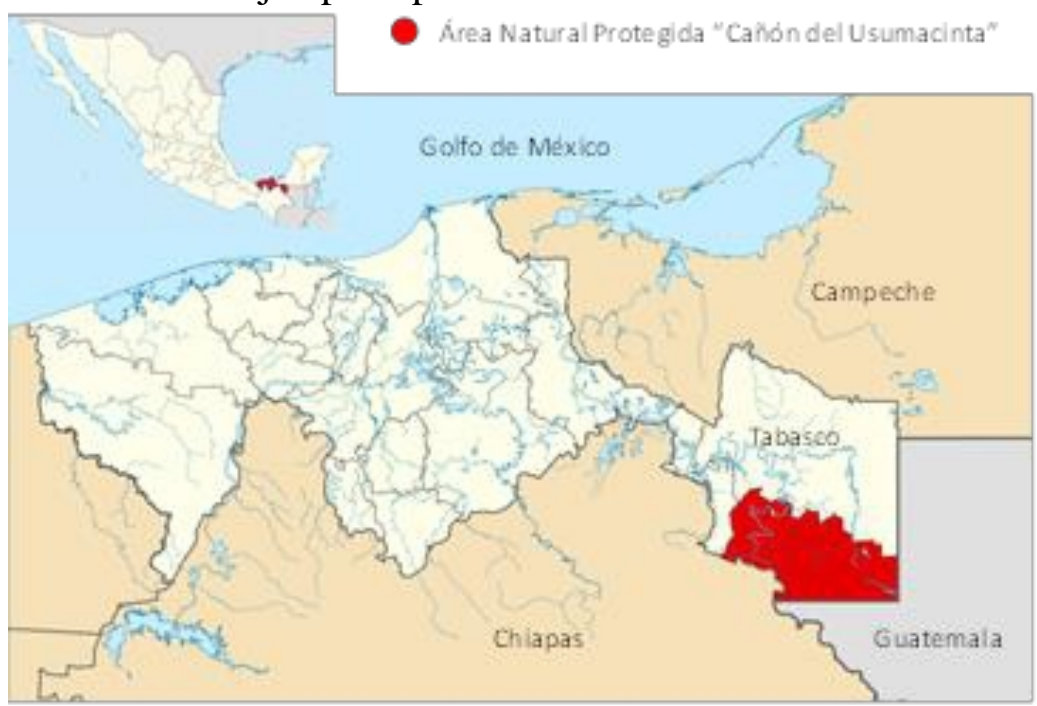

Figura 1. Ubicación de la Zona de Protección de Flora y Fauna Cañón del Usumacinta.

El secado de alimentos es una operación unitaria destinada a la eliminación de humedad mediante aplicación de calor. La reducción del contenido de humedad aumenta la vida útil de los alimentos ya que ocasiona un descenso de la actividad de agua $\left(a_{\mathrm{w}}\right)$, lo que inhibe el crecimiento microbiano y disminuye la velocidad de las reacciones deteriorantes. Además, la reducción de la masa y el volumen del producto favorece operaciones unitarias como empacado, embalaje, almacenamiento y transporte y, con ello, la diversidad en las presentaciones (Belén-Camacho et al., 2007).

Por razones económicas, ambientales y políticas, es necesario buscar otras fuentes alternativas de energía que resulten económicas, abundantes, limpias y que preserven el equilibrio ecológico. La energía proveniente del sol, del viento y de la tierra son opciones; sin embargo, la energía solar es el mejor candidato debido a que emite energía todo el tiempo al planeta. Todos los lugares reciben la misma energía, es abundante y gratuita, no es contaminante y no produce desechos radioactivos. Nadie puede aumentar su precio, no necesita cables o tanques para su transportación y es segura, ya que el sol es responsable de que en el planeta existan las condiciones adecuadas para la supervivencia de la vida humana, animal y vegetal (Nandwani, 2005). El secado solar se presenta como una alternativa en países tropicales en donde la incidencia de la energía del sol puede ser aprovechada para obtener alimentos deshidratados (Belén-Camacho et al., 2007). En este trabajo se ha 
tomado como caso de estudio el secado de chile jalapeño, debido a que es un producto agrícola de gran importancia para México, dado que ocupa el segundo lugar mundial como productor de esta hortaliza, con una producción de 1.8 millones de toneladas anuales (25\% de la cual se exporta), que equivalen a cerca de 150,000 ha sembradas (INEGI, 2005).

La aplicación de métodos sustentables para el procesamiento del chile jalapeño puede coadyuvar a la economía de las comunidades dentro del ANP al proporcionar valor agregado a los cultivos agrícolas, conservar los productos durante tiempos largos de almacenamiento y cubrir la demanda fuera de temporada. El objetivo de este trabajo fue evaluar la calidad física y microbiológica de la harina de chile jalapeño deshidratado en un secador solar tubular artesanal.

\section{Materiales y Métodos}

Área de estudio. El clima de Tabasco se define como cálido húmedo, caracterizándose por temperaturas elevadas, con un promedio anual mayor a los $26{ }^{\circ} \mathrm{C}$. La temperatura máxima promedio anual es de $32{ }^{\circ} \mathrm{C}$ y se presenta antes del inicio de la temporada de lluvias y del solsticio de verano. La temperatura mínima promedio anual es de $20{ }^{\circ} \mathrm{C}$ y se presenta en el mes de enero durante la temporada de invierno. La precipitación media mensual durante la temporada de lluvias es de 1,700 a 2,200 mm, presentando una humedad relativa promedio del $75 \%$ (Casas-Andreu et al., 2011).

Muestras. Se utilizaron chiles jalapeños de primera calidad de acuerdo a la clasificación de la NMX-FF-025-SCFI-2007, adquiridos en un supermercado local. De este lote se seleccionaron chiles con un grado de madurez de acuerdo a la clasificación de Sáenz y D’Alolio (2007), color verde uniforme, tamaño homogéneo y ausencia de daños físicos aparentes. Los frutos seleccionados se lavaron con agua potable y con ayuda de un cuchillo se realizaron los cortes de forma manual: cortes en rodajas y juliana.

Secador solar. Para el secado se construyó un equipo solar tubular con dimensiones de $0.5 \mathrm{~m}$ de ancho, $2.0 \mathrm{~m}$ de largo y $1.5 \mathrm{~m}$ de alto, como se muestra en la Figura 2. 


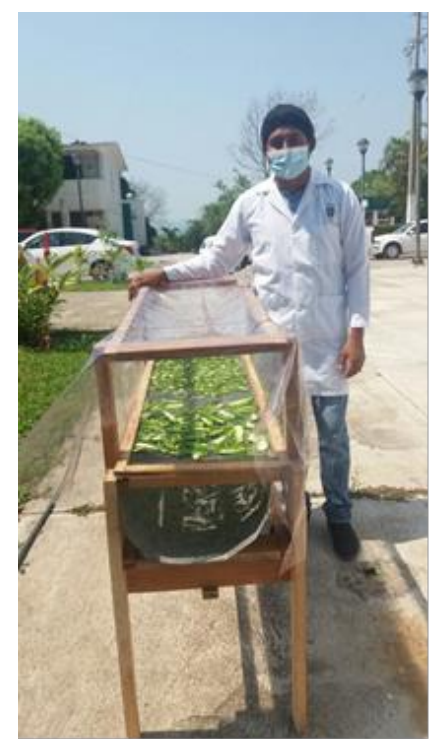

Figura 2. Prototipo artesanal del secador solar tubular.

El prototipo se elaboró empleando madera, lámina de zinc y plástico transparente, de acuerdo al modelo propuesto por Oliveros-Tascón et al. (2006), con modificaciones. El plástico transparente evita la precipitación de partículas de polvo durante el secado, con la finalidad de disminuir la contaminación microbiológica por partículas de polvo suspendidas en el aire, mientras que el canal de la lámina permite el flujo del aire caliente hacia los extremos.

Proceso de secado. Se evaluaron tres técnicas de secado: solar directo, en estufa eléctrica y en prototipo experimental.

i) Secado solar directo. El secado de los chiles se realizó directamente al sol, mediante el método de secado por conducción, empleando una lámina de zinc. Este proceso parte del principio en el cual la materia prima entra en contacto térmico directo con una superficie caliente y la mayor parte de la transferencia de calor se produce por conducción (Marín et al., 2011).

ii) Secado en estufa de convección. Se realizó de acuerdo a la metodología propuesta por García et al. (2013) empleando una estufa de secado convencional (Binder ${ }^{\circledR}$ ) a una temperatura de $70{ }^{\circ} \mathrm{C}$, hasta obtener un peso constante.

iii) Secado en prototipo experimental. El secado solar se presenta como una alternativa en países tropicales en donde la incidencia de la energía solar puede ser aprovechada para obtener alimentos deshidratados, minimizando el consumo de otras fuentes de energía más costosas como los combustibles o la energía eléctrica, permitiendo secar alimentos al ambiente con un proceso menos contaminante y con menos gastos operativos (Belén- 
Camacho et al., 2007). Las rodajas y julianas de chile jalapeño se colocaron directamente sobre la malla del secador. La temperatura en el interior del secador fue de $43{ }^{\circ} \mathrm{C} \pm 2{ }^{\circ} \mathrm{C}$ y en el exterior de $41^{\circ} \mathrm{C} \pm 1{ }^{\circ} \mathrm{C}$, variando con la hora del día.

Se evaluó el peso cada hora para determinar la pérdida de humedad. Para el secado se establecieron cargas de $500 \mathrm{~g}$ de material en forma de capa, exponiendo a la radiación solar por el tiempo establecido, en el lapso comprendido entre 08:00 am y 6:00pm, empleando nueve horas diarias durante dos días soleados. El producto seco fue colocado en bolsas de polietileno de capacidad de $1,000 \mathrm{~g}$ y almacenados a temperatura ambiente.

Elaboración de harina. Posterior al secado de los chiles jalapeños se realizó la molienda y almacenamiento.

i) Molienda. Se realizó de acuerdo a lo recomendado por Cabrera et al. (2007), empleando un molino manual (Estrella $\left.{ }^{\circledR}\right)$. El objetivo de la molienda, es la reducción del tamaño de los sólidos por impacto, abrasión o fricción, hasta partículas menores a $1 \mathrm{~mm}$ (Sala \& Barroeta, 2003), que se satisfagan las condiciones y especificaciones para el producto a elaborar. La molienda es de gran importancia, porque una buena apariencia granular incidirá favorablemente en la aceptación del producto en el mercado.

ii) Almacenado. Se almacenó en recipientes de vidrio, cerrados herméticamente y mantenidos a temperatura ambiente $\left(22-28^{\circ} \mathrm{C}\right)$. El tiempo de almacenamiento esperado fue de seis meses, de acuerdo a las recomendaciones de Cortés (2010), quien recomienda este tiempo para una temperatura cercana a los $27^{\circ} \mathrm{C}$.

Análisis microbiológicos. Se evaluó la concentración de los microorganismos mesófilos aerobios (Agar para cuenta estándar, Bioxon®), coliformes totales (Agar de Bilis y Rojo Violeta, Bioxon $\left.{ }^{\circledR}\right)$, Staphylococcus aureus (Agar de Sal y Manitol, Bioxon ${ }^{\circledR}$ ) y bacterias lácticas totales (Agar de Man Rogosa y Sharpe, Difco®) por el método propuesto por Corona y Jiménez (2004), los aerobios se incubaron a $37{ }^{\circ} \mathrm{C}$ durante $24 \mathrm{~h}$, y los anaerobios, durante $48 \mathrm{~h}$ en bolsa anaerobia (Rosenblatt \& Stewart, 1975).

Color. Se evaluó sensorialmente el cambio de coloración, mediante la técnica propuesta por Costell (2005), para establecer comparaciones con un patrón. Con esta técnica pueden evaluarse diversos atributos, se deciden los atributos sensoriales más importantes en el producto y se evalúan, en todos ellos, las magnitudes de las diferencias respecto al estándar. En este estudio se evaluó la diferencia en color con una escala de diez valores desde "no hay diferencia" hasta "muy diferente", tomando como referencia el color verde del chile sin procesar. 


\section{Resultados}

Se evaluó el secado solar como una estrategia sostenible para la conservación de chile jalapeño cultivado por los habitantes de un ANP. El objetivo fue comparar la calidad física y microbiológica de harina de chile jalapeño producida mediante tres procesos de deshidratación: secador solar, en estufa convencional y en un prototipo solar. La Figura 3 muestra la curva de secado en las tres técnicas evaluadas.

Para el secado directo se emplearon $18 \mathrm{~h}$ para obtener la eliminación del agua hasta obtener un peso constante. El uso del secador redujo $4 \mathrm{~h}$ de exposición solar para obtener un producto deshidratado, de 18 a 14 h. Además, con el secado en rodajas se obtuvo una deshidratación adecuada empleando solamente ocho horas de exposición, lo que permite obtener harina de chile jalapeño empleando sólo un día soleado, duplicando la producción.

La Figura 4 muestra los cambios en la coloración de la harina. Se obtuvo una harina con una coloración oscura, perdiendo el color verde característico del chile jalapeño. El color obtenido con el secado en estufa eléctrica fue más oscuro que el obtenido con el secador solar; probablemente el oscurecimiento está asociado a la temperatura empleada en la estufa eléctrica, de $70{ }^{\circ} \mathrm{C}$, mientras que el secador solar, la temperatura fue menor, aunque el tiempo de exposición fue mayor.

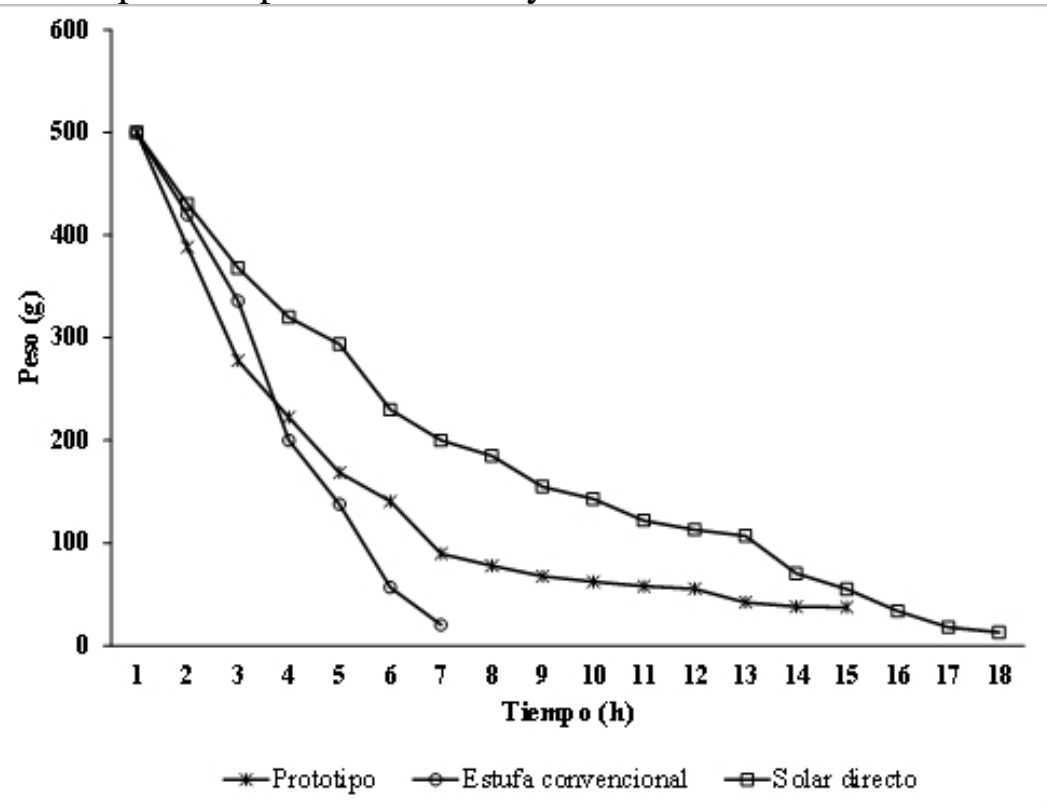

Figura 3. Tiempo de secado de chile jalapeño empleando tres técnicas de deshidratación.

Al comparar las ocho horas requeridas en el secador solar con las siete de la estufa eléctrica, el proceso de deshidratación de chiles en el trópico es una opción muy ventajosa para el secado solar, ya que puede obtenerse un 
producto con una concentración adecuada de humedad que permita conservarlo por mucho tiempo sin invertir energía eléctrica. Este proceso permitirá realizar una transferencia exitosa en comunidades del ANP, quienes podrán realizar el secado en condiciones muy amigables con el medio ambiente.

El Cuadro 1 muestra la concentración de microorganismos indicadores las harinas procesadas empleando las tres técnicas de secado. Los resultados muestran que la harina producida en la estufa eléctrica presentó la menor carga microbiana. Al comparar el secado solar directo y el secador tubular experimental, la concentración bacteriana fue menor al emplear el modelo de secador. La diferencia entre estas dos técnicas puede deberse a la cubierta plástica del secador, que impidió la sedimentación de partículas presentes en el ambiente. Lo anterior permitió obtener un producto con la calidad microbiológica permitida por la norma mexicana para las harinas destinadas al consumo humano.

Cuadro 1. Concentración de microorganismos en harina de chile jalapeño, por tres técnicas.

\begin{tabular}{lcccc}
\hline Microorganismos & $\begin{array}{c}\text { Estufa } \\
\text { (UFC/g) }\end{array}$ & $\begin{array}{c}\text { Sol directo } \\
\text { (UFC/g) }\end{array}$ & $\begin{array}{c}\text { Secador tubular } \\
\text { (UFC/g) }\end{array}$ & $\begin{array}{c}\text { NOM-247-SSA1- } \\
\text { 2008 (UFC/g) }\end{array}$ \\
\hline Coliformes totales & 0 & 0 & 25 & $<30$ \\
Staphylococcus aureus & 0 & 83 & 62 & - \\
Mesófilos aerobios & 0 & 93 & 57 & 10000 \\
Bacterias lácticas & 0 & 0 & 0 & - \\
\hline
\end{tabular}

\section{Discusión}

El secado es un proceso esencial en la preservación de los productos de origen agrícola. El primer método de secado desarrollado por el hombre consistió en la exposición directa del producto al sol. Si bien, el secado con la quema de madera genera un producto en menor tiempo tienen la desventaja de los daños que causan al ambiente vía deforestación y contaminación del aire (Hernández et al., 2008).

Oliveros-Tascón (2006) mostraron que es posible emplear materiales propios de la región para la construcción del secador combinado con material plástico transparente que permita el paso de la luz, el cual resultó ser de fácil manejo y económico. Una de las desventajas del material plástico es la contaminación que generaría después de su vida útil. Sin embargo, el material plástico puede reciclarse o reutilizarse cuando no permita el paso de luz solar. Este tipo de secadores se recomienda para regiones donde no es posible utilizar energía eléctrica o energía combustible de árboles.

El tiempo de secado promedio en el deshidratador solar experimental fue menor que el tiempo del secado solar directo. De igual manera, al comparar el tiempo de secado con la estufa eléctrica, el tipo de corte hizo que en juliana disminuyera el tiempo de secado. En trabajos de secado solar realizados por Hernández et al. (2014) el tiempo aproximado para deshidratar alimentos 
como papaya, chile habanero, zanahoria y nopal fue $10 \mathrm{~h}$ aproximadamente, a una temperatura de entre $47 \mathrm{y} 48{ }^{\circ} \mathrm{C}$. Aunque la temperatura en el secador solar tubular fue menor, el tiempo de secado también disminuyó, en relación al reportado.

Hernández et al. (2010) reportaron que se puede lograr un secado de calidad del chile habanero con temperaturas a partir de los $50{ }^{\circ} \mathrm{C}$, cuando se corta en rodajas de espesor de entre 0.3 y $1.3 \mathrm{~cm}$. Los resultados obtenidos muestran que se requieren de $12 \mathrm{~h}$ de tiempo de operación efectiva del secador solar para alcanzar un contenido de humedad final de $5 \%$, en tanto que en un secador convectivo convencional se requieren entre $14 \mathrm{~h} \mathrm{y} 6 \mathrm{~h}$ con temperaturas entre 50 y $55{ }^{\circ} \mathrm{C}$ para obtener un contenido de humedad semejante.

En relación a la calidad microbiológica, la harina de chile jalapeño se encontró dentro de los valores permitidos por Norma Oficial Mexicana NOM247-SSA1-2008. Alimentos a base de: cereales, semillas comestibles, de harinas, sémolas o semolinas o sus mezclas, donde los valores obtenidos en la harina de chile jalapeño se encuentran muy por debajo de los valores establecidos para harinas de trigo.

En un estudio realizado por Zavaleta-Avejar et al. (2008) evaluaron el secado de chile habanero ( $C$. chinense Jaq.) en estufa y en un secador solar. Aunque los chiles deshidratados en estufa presentaron mejores características microbiológicas, el secado solar es una alternativa de procesamiento adecuada para los productores agrícolas de bajo nivel socioeconómico, por no requerir de personal especializado y con requerimientos mínimos de infraestructura. En secado solar de jitomates, Mezquitillo-Bocanegra et al. (2014) obtuvieron producto deshidratado sin cambio considerable en el color, tomando como referencia el producto sin deshidratar, empleando temperaturas que van desde $45^{\circ} \mathrm{C}$ hasta $55^{\circ} \mathrm{C}$. En el secado del chile jalapeño, se observó un cambio en la coloración verde por coloración marrón, sin embargo, este cambio fue menor en el secador solar. La temperatura del secador solar experimental se encontró entre 41 y $45^{\circ} \mathrm{C}$. 

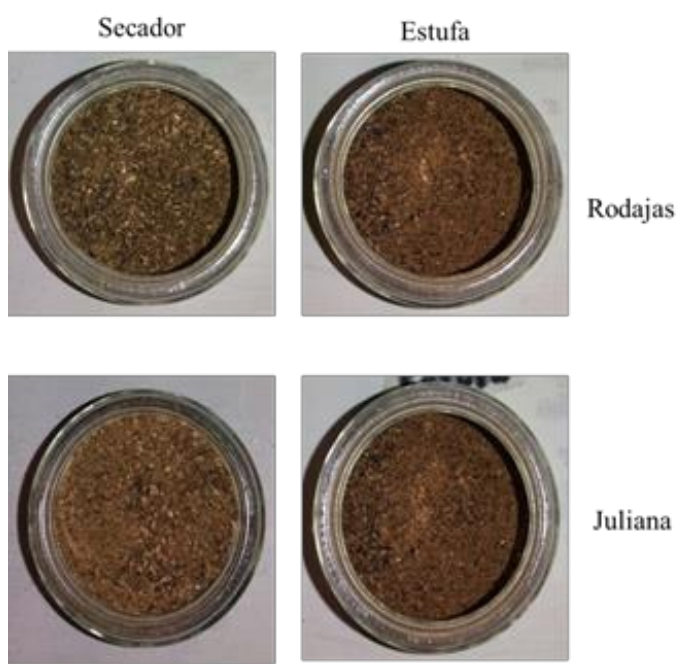

Figura 4. Coloración de harina de chile jalapeño en secador solar y estufa eléctrica.

En algunos modelos de secador solar, se ha reportado variaciones en las temperaturas dentro del secador, como el evaluado por MezquitilloBocanegra et al. (2014). Estas variaciones en la temperatura se deben a cambios provocados por el modelo de secador solar. En el secador evaluado en este estudio, la temperatura se mantuvo uniforme debido a la disposición de la lámina de zinc, así como al tubo central, que contribuye a una distribución uniforme del calor ya un flujo de aire adecuado.

Los factores claves para un buen secado son: aire a una temperatura entre 40 y $70{ }^{\circ} \mathrm{C}$, bajo contenido de humedad y movimiento constante. Para eliminar la humedad de los alimentos, es necesario que el aire que pasa por los productos este en constante movimiento y renovación. Esta ventilación se puede lograr en forma natural gracias al efecto chimenea o en forma forzada mediante ventiladores, dependiendo del modelo del secadero. Para obtener un buen secado, los productos tienen que ser colocados de tal forma que haya suficiente espacio entre las partes que los componen (Almada et al., 2005).

Las tecnologías de deshidratado solar desarrolladas están orientadas a soluciones económicas, como apoyo a pequeños y medianos productores, compatibles con el medio ambiente, buscando la eficiencia energética y utilizando materiales de fácil acceso. Los módulos deshidratadores solares han demostrado ser una alternativa muy apropiada a las necesidades requeridas por las Pymes agrícolas, con un diseño: unimodular, transportable, versátil, y sustentable con el medio ambiente, factores que lo hacen una alternativa muy atractiva al momento de agregar valor a la producción agrícola (Espinoza, 2016).

La difusión y transferencia de la tecnología desarrollada a pequeños productores rurales, contribuye a mejorar su calidad de vida al posibilitarles 
ingresos adicionales por diversificación productiva, además de contribuir al aprovechamiento sostenible del bosque como fuente de recursos alimenticios. La aplicación de métodos sustentables permitirá mejorar el ingreso económico de los habitantes del Área Natural Protegida, proporcionar valor agregado a sus cultivos, conservar sus productos para cubrir necesidades fuera de temporada y mejorar la relación con su medio ambiente.

\section{Conclusión}

El secado solar es una alternativa para la conservación de alimentos mediante la deshidratación. En este trabajo fue posible obtener harina de chile jalapeño mediante los tres métodos de secado comparados (deshidratación solar directa, prototipo experimental y estufa convencional). Se observó que el periodo de secado fue mayor en el prototipo propuesto; sin embargo, se conservó mejor el color de las muestras en comparación con el método convencional. En cuanto a la calidad microbiológica, con el método convencional y el prototipo experimental se obtuvo un producto con la concentración microbiana requerida para el consumo humano, al presentar valores dentro de los límites permitidos por la norma mexicana. La aplicación de métodos sustentables puede coadyuvar a la economía de las comunidades que habitan en el Área Natural Protegida. De igual manera, apoyará el fortalecimiento de la cadena de valor de los productos agrícolas, así como a mantener una mejor relación con el medio ambiente.

\section{References:}

1. Almada, M., Cáceres, M., Machain-Singer, M. \& Pulfer, J. (2005). Guía de uso de secaderos solares para frutas, legumbres, hortalizas, plantas medicinales y carnes. Fundación Celestina Pérez de Almada. Asunción. 42 p.

2. Belén-Camacho, D., Román, J., García, D., Moreno-Álvarez, M., Medina, C. \& Ojeda, C. (2007). Efecto del secado solar en los contenidos de humedad, carbohidratos, carotenoides totales e índices de peróxidos del mesocarpio de la palma Coroba (Attalea spp.). Interciencia, 32(4):257-261.

3. Cabrera, O., Chacón, P., López, C., Tapia, M. \& Villalva, M. (2007). Harina de pescado. Disponible en http://harinadepescadogrupo2.blogspot.mx/2007_06_01_archive.html.

4. Casas-Andreu, Gustavo, Barrios-Quiroz, Gabriel, \& Macip-Ríos, Rodrigo. (2011). Reproducción en cautiverio de Crocodylus moreletii en Tabasco, México. Revista mexicana de biodiversidad, 82(1):261273. Recuperado en 19 de agosto de 2017, de http://www.scielo.org.mx/scielo.php?script=sci_arttext\&pid=S1870$34532011000100023 \& \operatorname{lng}=$ es\&tlng=es. 
5. Comisión Nacional de Áreas Naturales Protegidas, CONANP. (2016). Áreas naturales protegidas de México. Disponible en http://www.conanp.gob.mx/regionales/

6. Corona, A. \& Jiménez, R. (2004). Comparación de dos métodos de siembra para el recuento de microorganismos en muestras con alta concentración microbiana. Revista de la Facultad de Ingeniería Química. 40:3-7.

7. Cortés, V. (2010). Sustitución parcial de harina de pescado (Plecostomus spp.) por harina de lombriz (Eisenia foetida) en alimento para bagre de canal (Ictalurus punctatus). Tesis de Maestría en Ciencias en Producción Agrícola Sustentable, Instituto Politécnico Nacional, CIIDIR, Michoacán. Jiquilpan, Michoacán. 81 p.

8. Costell, E. (2005). El análisis sensorial en el control y aseguramiento de la calidad de los alimentos: una posibilidad real. CTC Alimentación. 23:10-17.

9. Espinoza, J. (2016). Innovación en el deshidratado solar. Ingeniare, Revista Chilena de Ingeniería. 24:72-80.

10. García, A., Muñiz, S., Hernández, A., González, L. \& Fernández, D. (2013). Análisis comparativo de la cinética de deshidratación osmótica y por flujo de aire caliente de la piña (Ananas comosus, variedad Cayena lisa). Revista Ciencias Técnicas Agropecuarias. 22(1):62-69.

11. García-Morales, R., Gordillo-Chávez, E., Valdez-Leal, J. \& PachecoFigueroa, C. (2014). Las áreas naturales protegidas y su papel en la conservación de los murciélagos del estado de Tabasco, México. Therya. 5(3):725-736.

12. Hernández, J., Quinto, P., Cuevas, J., Acosta, R. \& Aguilar J. (2008). Estudio del secado de Capsicum annuum L. a través del modelo de Luikov. Caos Conciencia. 1:21-30.

13. Hernández, J., Martínez, O., Quinto, P., Cuevas, J., Acosta, R., \& Aguilar, J. (2010). Secado de chile habanero con energía solar. Revista Iberoamericana de Tecnología Postcosecha. 10(2):120-127.

14. Hernández, J., Flores, F., Acosta, R. \& Barbosa, G. (2014). Secado solar de productos agrícolas. Caos Conciencia. 8(1):25-34.

15. Instituto Nacional de Estadística, Geografía e Informática, INEGI. (2005). Anuario Estadístico de los Estados Unidos Mexicanos. Edición 2005. pp 56-59.

16. Marín, D., Alcocer, M., Salazar, N. \& Bernal, J. (2011). Calidad de la harina de arracacha (Arracacia xanthorriza Bancroft) a partir del método de secado por conducción. Revista de Investigación Agraria y Ambiental RIAA. 2(1):23-28.

17. Mezquitillo-Bocanegra, C., Ruiz-Camacho, B. \& Martínez Álvarez, O. (2014). Construcción y evaluación de un secador solar indirecto para 
el deshidratado de tomate saladette. Química Hoy Chemistry Science. 4(1)12-16.

18. Nandwani, S. (2005). Energía solar, conceptos básicos y su utilización. Laboratorio de Energía Solar, Departamento de Física, Universidad Nacional, Heredia, Costa Rica. 26 p.

19. Norma Mexicana NMX-FF-025-SCFI-2007. Productos alimenticios no industrializados para consumo humano. Chile fresco (Capsicum spp.). Especificaciones

20. Norma Oficial Mexicana NOM-247-SSA1-2008. Productos y servicios. Cereales y sus productos. Cereales, harinas de cereales, sémolas o semolinas. Alimentos a base de: cereales, semillas comestibles, de harinas, sémolas o semolinas o sus mezclas. Productos de panificación. Disposiciones y especificaciones sanitarias y nutrimentales. Métodos de prueba.

21. Oliveros-Tascón, C., Ramírez-Gómez, C., Zans-Uribe, J. \& PeñuelaMartínez, A. (2006). Secador solar de túnel para café pergamino. Avances Técnicos Cenicafé. 353, 1-8.

22. Rosenblatt, J. \& Stewart, P. (1975). Anaerobic bag culture method. Journal of Clinical Microbiology. 1(6):527:530.

23. Sáenz, A. \& D'Alolio, O. (2007). Identificación de pérdidas poscosecha de chile jalapeño [Capsicum annuum L. cultivar mitla] para uso agroindustrial. Consejo Nacional de Producción. San José (Costa Rica).pp 5.

24. Sala, R. \& Barroeta, A. (2003). Manual de microscopía de piensos. Colección Materials 122. Universitat Autònoma de Barcelona, Servei de Publicacions. Barcelona. pp 100.

25. Zavaleta-Avejar, L., Pérez-Orozco, J. \& Herrera-Rodríguez, F. (2008). En Memorias del V Congreso Internacional de Ingeniería Bioquímica. México, D. F. 